\title{
Corrigendum
}

\section{Global oral public health - The current situation and recent developments}

\author{
Kenneth A. Eaton
}

European Association of Dental Public Health, Old Saddlers, Kempe's Corner, Canterbury Road, Boughton Aliph, Ashford, Kent, TN25 4 EW, UK.

Journal of Public Health Policy (20I2) 33, 492. doi:I0.1057/jphp.20I2.38

Correction to: Journal of Public Health Policy (2012) 33, 382-386. doi: I0.1057/jphp.20I 2.22

The author's contact email was incorrect in the original version; it should read kenneth.a.eaton@btinternet.com. 